\title{
The effects of carbon dioxide- and neodymium-YAG lasers on the central and peripheral nervous systems, and cerebral blood vessels
}

\author{
GF WALTER, ${ }^{*}$ PW ASCHER, $\dagger$ E INGOLITSCH \\ From the Laboratory of Neuropathology of the Institute of Pathological Anatomy* and Clinic of \\ Neurosurgery, $\dagger$ University of Graz, Austria
}

SUMMARY The morphological reaction of brain and peripheral nerve tissue and of cerebral blood vessels to the application of two types of laser-beams is described. Lasers as cutting instruments produce smoother cut surfaces and provoke less bleeding than conventional techniques. There is almost no focal oedema. Their effects are limited to clearly determinable zones. Scar formation after laser cutting is minimised. With defocused laser-beams a photo-coagulation of tumours, for example, highly vascularised tumours or pituitary adenomas, is possible. Small arteries may be cut without bleeding. Peripheral nerves cut by use of lasers do not develop neuromas. The results of the laser technique are compared with those of conventional methods.

The use of the laser in surgery is well established. ${ }^{1}$ First experimental studies on its effects on the nervous system ${ }^{2-7}$ revealed various technical problems while still showing the possibility of the application of the laser as a neurosurgical instrument. Ascher ${ }^{8}$ introduced the carbon dioxide laser as a useful instrument in neurosurgery.

\section{Technical background}

A laser (acronym for "light amplification by stimulated emission of radiation") is a high-intensity light source which emits an almost parallel electromagnetic beam of given wavelength. The beam is generated in an atomic or molecular system by means of quantum-optical processes. The two laser types used for the present report are the carbon dioxide laser $\left(\mathrm{CO}_{2}\right.$-laser) and the neodymiumYAG laser (Nd-YAG laser). The $\mathrm{CO}_{2}$-laser (invisible, middle infrared, $\lambda=10600 \mathrm{~nm}$, maximum power output about $50 \mathrm{~W}$ ) is a molecular gas laser in which inversion occurs in excited $\mathrm{CO}_{2}$ molecules. The Nd-YAG laser (invisible, near infrared, $\lambda=1060 \mathrm{~nm}$, maximum power output about $100 \mathrm{~W}$ ) is a solid-state laser in which the amplifying medium consists of neodymium-ions in an

Address for reprint requests: Doz. Dr.med. Dr.phil. GF Walter, Laboratorium für Neuropathologie, Institut für Pathologische Anatomie der Karl-Franzens-Universităt Graz Auenbruggerplatz 25 A-8036 Graz/Austria.

Received 28 December 1982 and in final revised form 20 October 1983.

Accepted 5 July 1983 yttrium-aluminium-garnet lattice. The visible ruby- and argon-lasers which are mainly used in ophthalmology are less suitable in neurosurgery. Therefore, the invisible $\mathrm{CO}_{2}-$ and Nd-YAG lasers were adapted with a helium-neon pilot light laser necessary for the successful use as a neurosurgical instrument. The effect of the laser beam depends on three variable factors: the power output, the density of the beam (focused or defocused), and the exposure time.

\section{Material and methods}

Experimental studies in animal brains (Wistar rats, rabbits and cats) were performed to gain surgical skill. The results were not transferable to the human brain with a different thickness of the cortex and different blood flow. As soon as the laser apparatus was technically mastered, morphological studies on human brain tissue were started. In 11 adult tumour patients, stab incisions by the $\mathrm{CO}_{2}$-laser with changing parameters for the power output and the exposure time were produced in normal cortex and white matter adjacent to the tumour. In all these cases, a $125 \mathrm{~mm}$ lens strictly in focus was used. The output energy varied from 10 to $50 \mathrm{~W}$, the exposure time from 0.01 to $1 \mathrm{~s}$. At the same time and in the same patients, stab incisions by the diathermy-needle (diameter $0.6 \mathrm{~mm}$, energy approximately $30 \mathrm{~W}$ ) were produced. After removal of the cortex and white matter cylinders, the incision depths under all changing parameters were measured in histological sections. In 24 rabbits, a fascicle of the sciatic nerve was dissected either by use of the $\mathrm{CO}_{2}$-laser or by use of a microscissor. The sciatic nerves of four rabbits were histologically examined.

After the end of the experimental studies, the $\mathrm{CO}_{2}$-laser 
was used in 529 human cases (since 1976), and Nd-YAG laser in 61 human cases (since 1981) for neurosurgical operations (brain tumours, other intracranial interventions, spinal cord and peripheral nerve operations). The indications and clinical observations during surgery were summarised by Heppner and Ascher. ${ }^{9}$ An argon laser was experimentally tested but appeared to be less efficient.

For histological examination, tissue specimens were fixed in $10 \%$ neutral formaldehyde, embedded in paraffin and stained with $\mathrm{H}$ and E, PAS, Nissl's cresyl violet and Masson's trichrome. For demonstration of reticulin fibres, Gomori's impregnation was applied. Tissue from pituitary glands was investigated for its hormone-content (ACTH, GH, PRL, $\beta$-FSH, $\beta$-LH, $\beta$-TSH, $\alpha$-HCG, $\beta$-HCG, lipotropin) using the immunocytochemical PAP-technique. For transmission electron microscopy (TEM), specimens were fixed in $3 \%$ buffered glutaraldehyde, contrasted and post-fixed in $1 \%$ osmium tetroxide and embedded in Epon. Ultrathin sections were contrasted with uranyl acetate and lead citrate. Semithin sections were stained with toluidin-blue. For scanning electron microscopy (SEM),

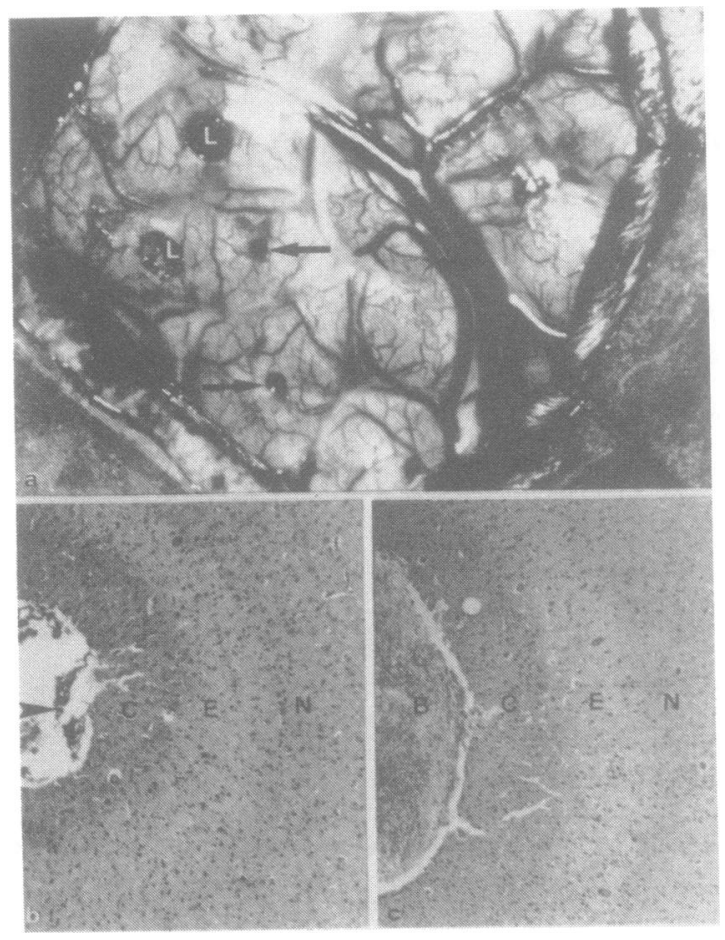

Fig 1 (a) Two focused $\mathrm{CO}_{2}$-laser incisions (arrows) and two focused Nd-YAG laser incisions $(L)$ in brain cortex. Operation microscope. (b) Focused $\mathrm{CO}_{2}$-laser incision with typical wall: carbonised tissue (arrow), photo-coagulation $(C)$, oedema $(E)$, normal tissue $(N)$. Note the absence of bleeding in the incision crater. (H and $E, \times 35$.) (c)

Diathermy-needle incision: there is bleeding in the incision crater $(B) . C=$ heat coagulation, $E=$ oedema, $N=$ normal tissue. ( $H$ and $E, \times 35$.) after fixation of the specimens with critical point drying, the cutting planes were sputtered with gold.

\section{Results}

The most important morphological results of experimental studies and clinical experiences are summarised.

THE LASER AND THE CENTRAL NERVOUS SYSTEM As a basis for the use of laser as a cutting instrument, cortical stab incisions of focused $\mathrm{CO}_{2}$-laser were compared with those of the diathermy needle. The laser incisions (fig 1a) were characterised by totally vaporised tissue without bleeding and a typi-

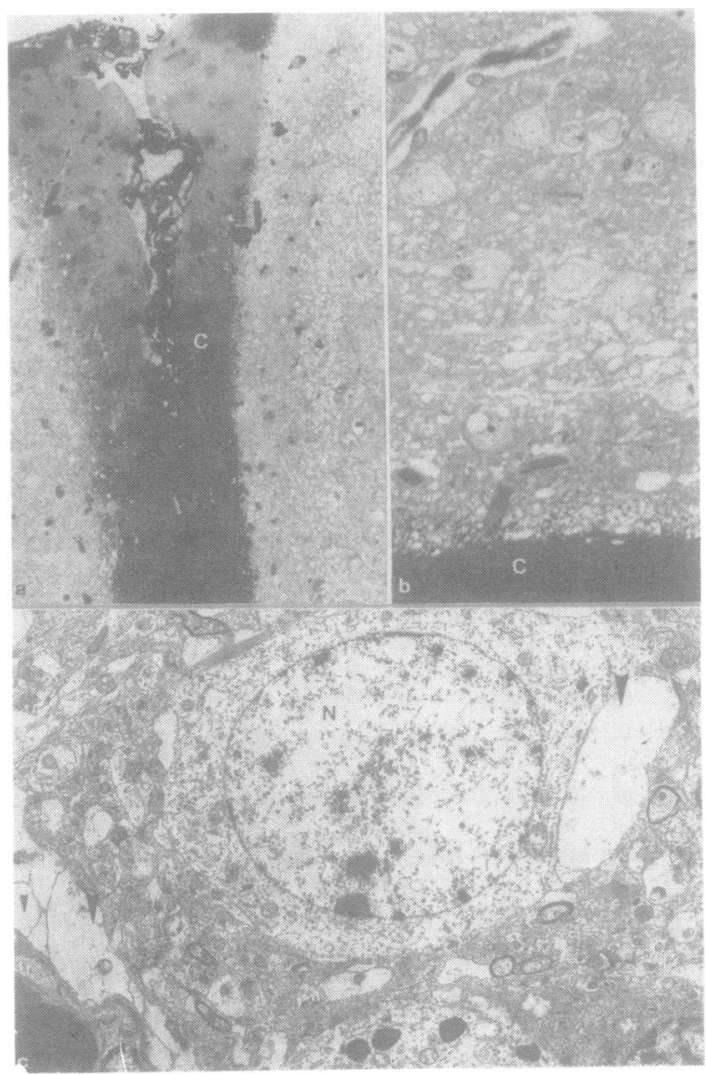

Fig 2 (a) Focused $\mathrm{CO}_{2}$-laser incision in brain cortex. Note the sharp edge of the photo-coagulation $(C)$. Semithin section (toluidin blue, $\times 17$.) (b) Cortical tissue next to the photo-coagulation $(C)$ showing vacuolar changes. The nerve cells are well preserved. Semithin section (toluidin blue, $\times 330$.) (c) Focused $\mathrm{CO}_{2}$-laser incision in brain cortex with relatively well preserved nerve cell $(N)$ in the oedema layer. Note the perivascular oedema and the vacuolar changes (arrows) next to the nerve cell. (Electron micrograph, $\times 48$ 000.) 
cal wall with an edge of carbonised, "smolten" tissue followed by well limited layers of photo-coagulation and oedema (figs $1 \mathrm{~b}$ and 2). In the layer of photocoagulation the nuclei were well stained, but the ultrastructure revealed the severe grade of necrosis. The oedema diminished in severity depending on the distance from the laser incision. In transition to normal brain tissue, the oedema was seen in perivascular astrocytic processes only. These results described for the $\mathrm{CO}_{2}$-laser were also valid for the Nd-YAG laser in focus. The depths and breadths of the incisions were dependent on the above mentioned variable factors: for a laser beam with a power output of $50 \mathrm{~W}$, a focal spot of $0.13 \mathrm{~mm}$ and an exposure time of 1 second a $450 \mu \mathrm{m}$ deep incision resulted which showed a wall of $20-30 \mu \mathrm{m}$ broad carbonisation, $100 \mu \mathrm{m}$ photo-coagulation and about $100 \mu \mathrm{m}$ broad oedema. These breadths should be considered as relative proportions owing to the shrinkage of tissue after fixation. The incision walls of the diathermy needle had a similar arrangement of heat-necrosis and oedema, but the lesion craters were filled with blood and residuals of combusted tissue (fig 1c). With scanning electron microscopy it could be seen that the cut surfaces after the use of a cold knife (suction tube) or the diathermy (Bovie) knife were irregular and rough and partly covered with blood (fig 3).

Neurosurgically, the focused laser beams were well suited for dissection of brain and spinal cord tumours. The possiblity of determining accurately the depth of tissue-vaporisation and the knowledge of the limitation of the photo-coagulation and oedema layers to fractions of millimeters permitted the removal of tumours of the brain stem successfully. ${ }^{9}$

The use of a defocused laser beam gave different results for the $\mathrm{CO}_{2}$ and the Nd-YAG lasers. The $\mathrm{CO}_{2}$-laser beam was entirely absorbed at the tissue surface, while the transmission of the Nd-YAG laser beam through tissue was much better and the beam was absorbed only at the depth of the tissue leaving the surface intact. The $\mathrm{CO}_{2}$-laser provoked a vaporisation of surface tissue followed by the typical layers, while the Nd-YAG laser provoked a necrotic "stopper" with only a minimal surface vaporisation (fig 4e).

Neurosurgically, the defocused $\mathrm{CO}_{2}$-laser was used for the vaporisation of brain wound surfaces after extirpation of glioblastomas in order to coagulate suspect remnants of tumour tissue. Although the rate of recurrence was not significantly influenced, there was very little postoperative glial scar formation (fig 4b).

In two cases of carcinomas with growth hormone receptors, the defocused Nd-YAG laser was used to

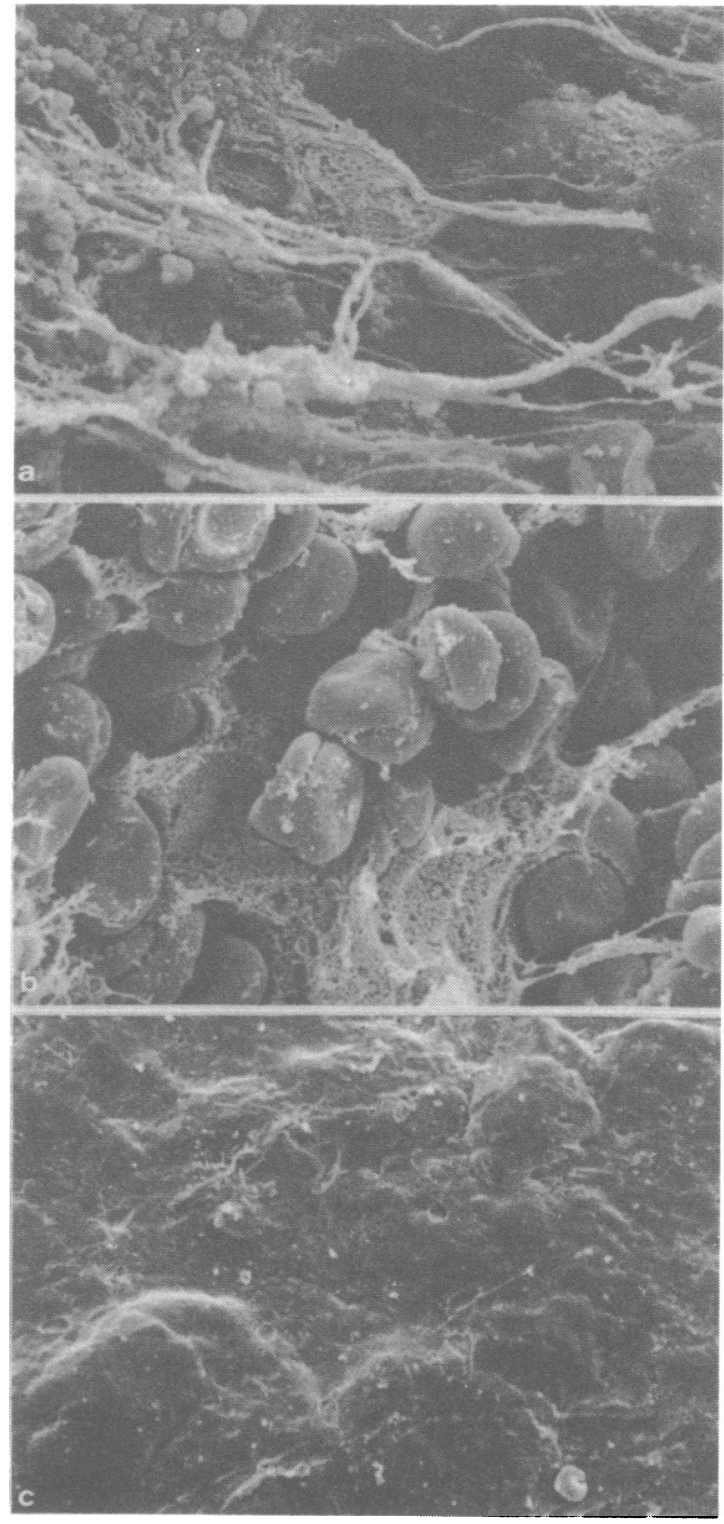

Fig 3 (a) Rough cut surface after use of a cold knife (suction tube). Scanning electron micrograph, $\times 1950$. (b) Rough cut surface covered with blood after use of a diathermy (Bovie) knife. Scanning electron micrograph, $\times 1950$. (c) Smooth cut surface without bleeding after use of the $\mathrm{CO}_{2}$-laser. Scanning electron micrograph, $\times 1950$.

coagulate the pituitary gland in situ. It appeared shrunken, the cell borders were blurred. Immunocytochemical investigations demonstrated the almost totally suppressed hormonal immunoreactivity. The clinical course of the patients 


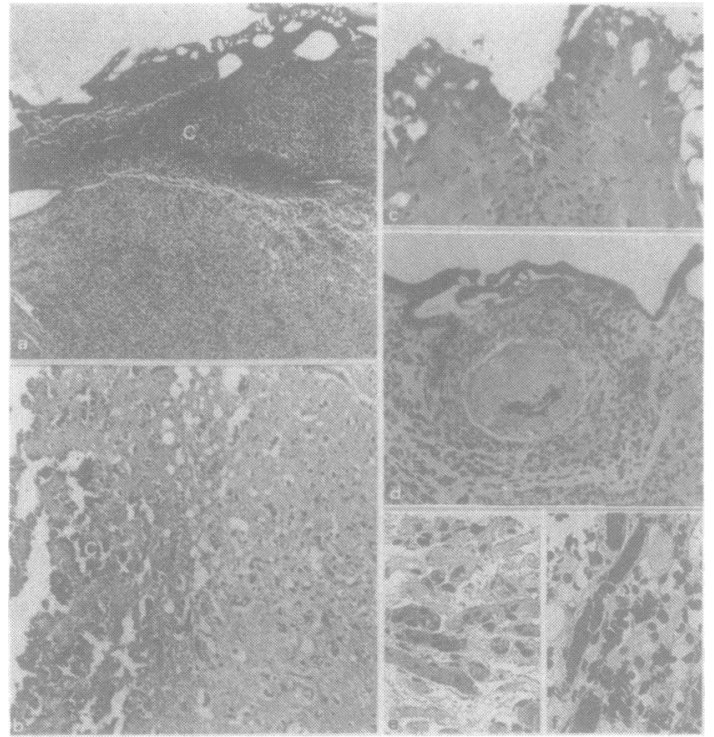

Fig 4 (a) Necrotic "stopper" produced by defocused $N d-Y A G$ laser with minimal surface vaporisation. Note the sharp edge of the photo-coagulation (C). (Masson trichrome, $\times 16$.) (b) Brain wound surface vaporisation by defocused $\mathrm{CO}_{2}$-laser after extirpation of a glioblastoma nine days before death from a pulmonary embolus. Note the minimal glial scar formation next to the photo-coagulation (C). ( $H$ and $E, \times 45$.) (c) Dissection of artery by focused $\mathrm{CO}_{2}$-laser with sealing of the vessel without bleeding. (van Gieson, $\times 45$.) (d) Coagulation of artery by defocused

$N d-Y A G$ laser with swollen vessel wall and narrow lumen. (Masson trichrome, $\times 45$.) (e) Coagulation of haemangiocytic meningioma by defocused Nd-YAG laser (Masson trichrome, $\times 80$.) (f) Part of the same haemangiocytic meningioma without laser radiation. (Masson trichrome, $\times 80$.)

improved, the size of the metastases decreased significantly after about three months. Long term results are not yet available.

\section{THE LASER AND CEREBRAL BLOOD VESSELS}

Dissection of arterioles and small arteries led to a contraction of the vessels. A sealing of the wound without bleeding was achieved in arteries with an outer diameter of $0.3-0.5 \mathrm{~mm}$ cut with the $\mathrm{CO}_{2-}$ laser and arteries with an outer diameter of $2 \mathrm{~mm}$ cut with the Nd-YAG laser (fig 4c).

With the defocused Nd-YAG laser it was possible to coagulate tumours including the vessels before removal in order to diminish the bleeding. Arterial vessel walls were swollen, the lumina narrow (fig 4d). This was very useful for the extirpation of arteriovenous malformations and of highly vascularised meningiomas, especially with sinus invasion

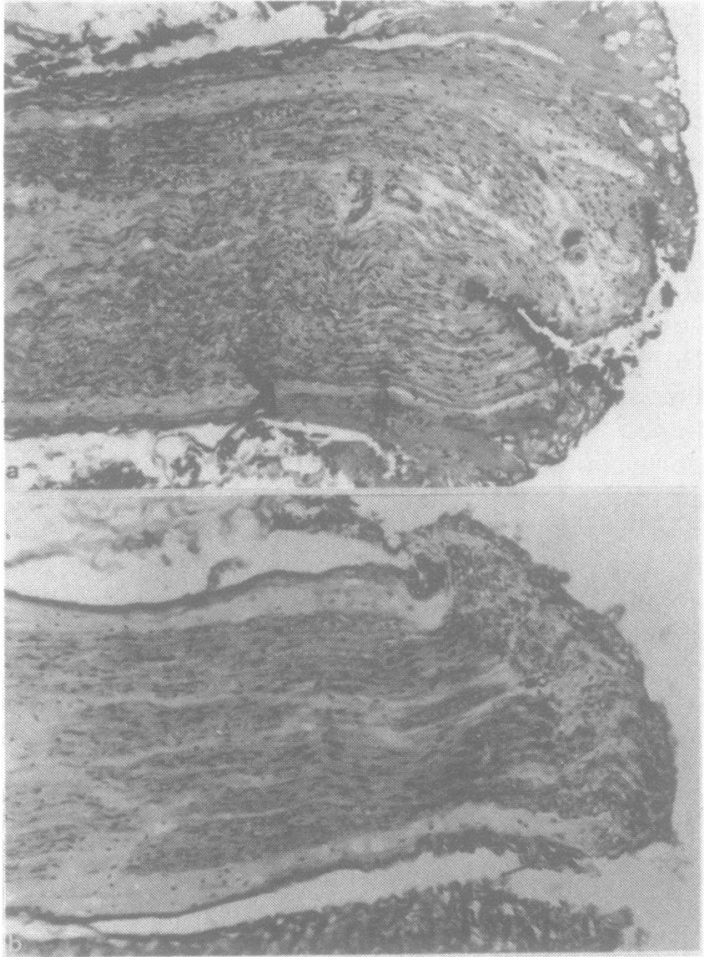

Fig 5 (a) Dissection of peripheral nerve by focused $\mathrm{CO}_{2}$-laser. Note the cut surface sealed by a thin layer of carbonised tissue. (van Gieson, $\times 40$.) (b) Dissection of peripheral nerve by micro-scissor. Note the cut surface with splitting of the tissue. (van Gieson, $\times 40$.)

(fig $4 \mathrm{e}$ and $\mathrm{f}$ ). The borders of the produced photocoagulation were distinct. The same results were achieved with gliomas.

\section{THE LASER AND THE PERIPHERAL NERVOUS SYSTEM}

The experimental dissection of a fascicle of the sciatic nerve in four rabbits either by the use of a laser beam or by the use of conventional instruments (micro-scissors) showed that laser cut surfaces were rather smooth with little damage to the endo- and perineurium, while conventional cutting produced splitting of the nerve fibres and severed the endoand perineurium. The surface after cutting with a micro-scissor did not give the "sealed" impression of the laser cut surface (fig 5). Scanning electron microscopic investigations demonstrated that the laser beam damaged the nerve fibre in a very limited small zone (fig 6). In long-term investigations of the nerves during the healing process after an interval of four months, neuroma formation was seen only in a group of 12 rabbits after conventional cutting and 


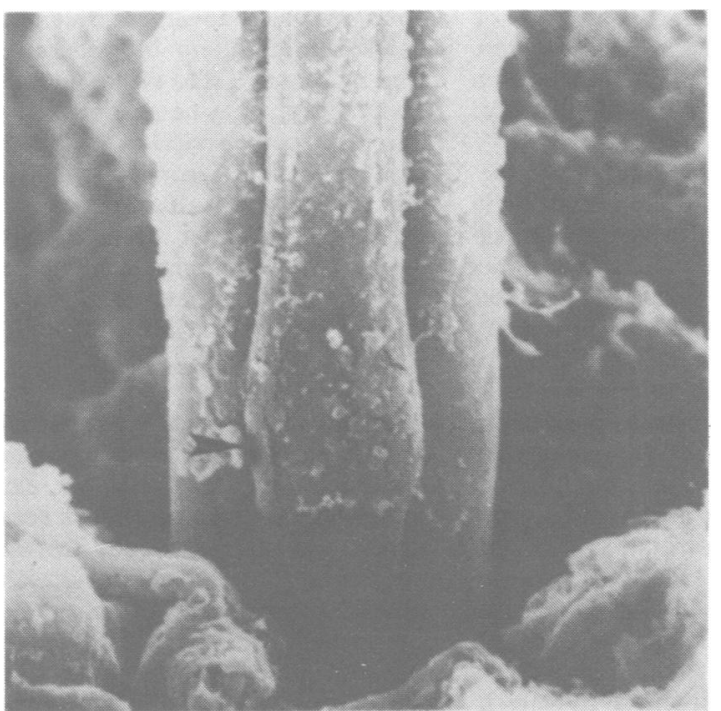

Fig 6 Initial effect of the $\mathrm{CO}_{2}$-laser on a fascicle of a peripheral nerve. The damage is limited to a narrow zone (arrow). Scanning electron micrograph, $\times 2200$.

never in the second group of 12 rabbits after laser dissection of the sciatic nerve.

In 22 patients with amputation stump neuromas the neuroma was exposed and the nerve transsected with the focused $\mathrm{CO}_{2}$-laser. To date no recurrence has been observed. ${ }^{9}$

\section{Discussion}

The basic advantage of the neurosurgical application of lasers is the employment of a non-touch technique which allows cutting without the slightest pulling of tissue. In combination with the accurate depth of the incisions, the possibility of operations arises in hitherto unaccessible regions such as the brain stem. There is no narrowing and no contamination of the operation field, and there is no disturbance of electrical monitoring.

It has been shown that laser incisions have smoother cut surfaces, a photo-energy action limited to determinable zones without damage of the surrounding tissue, and provoke less bleeding. The minimised scar formation is also advantageous for the avoidance of epileptogenic gliotic areas as well as amputation stump neuromas. On the other hand, the laser seems contraindicated in the field of plastic surgery as the nerve regeneration seems to be inhibited-at least under the experimental conditions investigated in the present study.

This paper and the report by Beck ${ }^{10}$ show that the defocused application of Nd-YAG laser produces photo-coagulation without vaporisation. This special kind of photo-coagulation of vascularised tumours or of pituitary glands or adenomas (in which the endocrine activity is eliminated and which are left in situ) may open up new possibilities in neurosurgical operations.

\section{References}

' Stellar S, Polanyi TG, Bredemeier HC. Lasers in surgery. In: Wolbarsht ML, ed. Laser Applications in Medicine and Biology, Vol. 2. New York: Plenum 1974:241-91.

${ }^{2}$ Stellar S. Effects of laser energy on brain and nerve tissue. Laser Focus 1965;1:3.

${ }^{3}$ Stellar S. A study of the effects of laser light on nervous tissue. Proc 3rd Int Congr Neurol Surg Amsterdam: Excerpta Medica 1966:542-51.

${ }^{4}$ Fox JL, Hayes JR, Stein MN, Green RC. Effects of laser radiation on intracranial structures. Proc 3rd Int Congr Neurol Surg Amsterdam: Excerpta Medica 1966:552-4.

${ }^{5}$ Fox JL, Stein MN, Hayes JR, Green RC. Effects of laser irradiation on the central nervous system. II. The intracranial explosion. J Neurol Neurosurg Psychiatry 1968;31:43-49.

- Lampert PW, Fox JL, Earle KM. Cerebral edema after laser radiation. J Neuropathol Exp Neurol 1966; 25:531-41.

${ }^{7}$ Stellar S, Polanyi TG, Bredemeier HC. Experimental studies with the carbon dioxide laser as a neurosurgical instrument. Med Biol Eng 1970;8:549-58.

${ }^{8}$ Ascher PW. Der $\mathrm{CO}_{2}$-Laser in der Neurochirurgie. Wien: Molden 1977.

${ }^{9}$ Heppner F, Ascher PW. The $\mathrm{CO}_{2}$-laser in neurosurgery. Int Adv Surg Oncol 1982;5:385-96.

${ }^{10}$ Beck OJ. Comparative studies on the use of lasers in neurosurgery. Paper presented at the 221st Symposium of the Medical Faculty of Munich University, 1981. 